\title{
ON REDUCING TO ZERO THE REMAINDER OF AN ASYMPTOTIC SERIES
}

\author{
J. E. DRUMMOND \\ (Received 23 September 1977; revised 2 February 1978)
}

\begin{abstract}
If a weighted Euler transformation is applied to the asymptotic series for $e^{z} E_{1}(z)$ the remainder can be expressed as an integral. Examination of this integral shows that for a transformation of given order the smallest term of the resulting series remains at approximately a constant distance from the start of the series. If, however, there is no restriction on the order of transformation the remainder may be decreased to zero by increasing the number of terms used, but if $z$ is close to the negative real axis the rate of decrease is small. A more general theorem for alternating real series and Taylor's series is also given.
\end{abstract}

Subject classification (Amer. Math. Soc. (MOS) 1970): 30 A 84, 41 A 60.

\section{Introduction}

An asymptotic power series is defined as a truncated power series, such as $\sum_{r=0}^{n}(-1)^{r} a_{r} / z^{r}$, for which the remainder is $O\left(1 / z^{n+1}\right)$. This means in practice that the sum is usually most accurate if we stop at the smallest term.

Sirovich (1971) shows that the Euler transformation may be used to continue analytically a power series outside its radius of convergence and mentions that a transformation by Shanks (1955) sometimes produces very striking increases in the rate of convergence of a series.

It is the purpose of this note to examine a transformation similar to but simpler than Shanks's and to show how it transforms the remainder of a particular asymptotic series. We proceed by first looking at a numerical example. The example is chosen because the remainder can be expressed as an integral. We may then consult tables of the integral for particular values and use the saddle point approximation to the integral for more general approximate properties. 


\section{The weighted Euler sum}

Let

where

$$
S(z)=S_{n}(z)+R_{n}(z)
$$

$$
S_{n}(z)=\sum_{r=0}^{n}(-1)^{r} a_{r} / z^{r}
$$

and $S(z)$ is a function of $z$ which has an asymptotic series $S_{n}(z)$ with a remainder $R_{n}(z)$.

We construct a weighted average of the asymptotic sums from $S_{m}(z)$ to $S_{n}(z)$ weighting each sum $S_{m+r}(z)(r=0,1, \ldots, n-m)$ with the binomial coefficient $\left(\begin{array}{c}n-m \\ r\end{array}\right)$ and $(-1)^{r}$ times the reciprocal of its last term, namely,

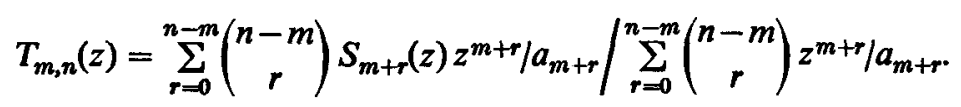

This transformation has been described by the author (1972) as a weighted Euler transformation and it has two further properties not mentioned in the previous paper:

(i) While any individual member of $T_{m, n}(z)$ may be calculated directly using equation (1), the whole table may be calculated using a recurrence relation as follows. Let

$$
P_{m, n}(z)=\sum_{r=0}^{n-m}\left(\begin{array}{c}
n-m \\
r
\end{array}\right) S_{m+r}(z) z^{m+r} / a_{m+r}
$$

and

$$
Q_{m, n}(z)=\sum_{r=0}^{n-m}\left(\begin{array}{c}
n-m \\
r
\end{array}\right) z^{m+r} / a_{m+r}
$$

hence

and

$$
P_{m, m}(z)=S_{m}(z) z^{m} / a_{m}, \quad Q_{m, m}(z)=z^{m} / a_{m}
$$

$$
P_{m, n}(z)=P_{m, n-1}(z)+P_{m+1, n}(z), \quad Q_{m, n}(z)=Q_{m, n-1}(z)+Q_{m+1, n}(z) .
$$

(ii) We may equally well use the reciprocals of the first term omitted in the weighted average. Thus, if we separate out the last term of each truncated series, $S_{m+r}(z)$, in the numerator of the right-hand side of equation (1) we obtain

$$
\sum_{r=0}^{n-m}\left(\begin{array}{c}
n-m \\
r
\end{array}\right)(-1)^{m+r}\left(\frac{a_{m+r}}{z^{m+r}}\right)\left(\frac{z_{m+r}}{a_{m+r}}\right)=(-1)^{m}(1-1)^{n-m}=0 \text { if } n>m .
$$

Hence we obtain an alternative expression

$$
T_{m, n}(z)=\sum_{r=0}^{n-m}\left(\begin{array}{c}
n-m \\
r
\end{array}\right) S_{m+r-1}(z) z^{m+r} / a_{m+r} / \sum_{r=0}^{n-m}\left(\begin{array}{c}
n-m \\
r
\end{array}\right) z^{m+r} / a_{m+r}
$$




\section{The remainder of a known asymptotic series}

If we integrate the exponential integral by parts $n$ times we obtain an asymptotic series whose remainder is expressed in closed form as another integral:

where

$$
\begin{aligned}
F_{1}(z)=e^{z} E_{1}(z) & =\int_{z}^{\infty} \frac{e^{z-x}}{x} d x \\
= & S_{n}(z)+(-1)^{n} F_{n+1}(z)
\end{aligned}
$$

$$
S_{n}(z)=\frac{0 !}{z}-\frac{1 !}{z^{2}}+\ldots+(-1)^{n-1} \frac{(n-1) !}{z^{n}}
$$

and

$$
F_{n}(z)=\int_{z}^{\infty} \frac{(n-1) ! e^{z-x}}{x^{n}} d x=\frac{(n-1) ! e^{z}}{z^{n-1}} E_{n}(z) .
$$

The integrals $E_{n}(z)$ are bounded except when $z=0$ or $-\infty$ and have been tabulated, for instance by Abramowitz (1964), for many real positive and complex values of $z$. When $z$ is complex the imaginary part of $F_{n}(z)$ has a discontinuity depending on whether the path of integration from $z$ to $\infty$ goes clockwise or anticlockwise round the origin. On the other hand, the value of $S_{n}(z)$ is unique. This contradiction is usually resolved by cutting the $z$ plane along the negative real axis.

If we construct the weighted Euler sum, $T_{m, n}(z)$, using equation (3) then the transformed remainder is given as

$$
\begin{aligned}
F_{1}(z)-T_{m, n}(z) & =\sum_{r=0}^{n-m}\left(\begin{array}{c}
n-m \\
r
\end{array}\right) \frac{z^{m+r}}{(m+r-1) !}(-1)^{m+r} F_{m+r}(z) / \sum_{r=0}^{n-m}\left(\begin{array}{c}
n-m \\
r
\end{array}\right) \frac{z^{m+r}}{(m+r-1) !} \\
& =(-1)^{m-1} \int_{z}^{\infty}\left(\frac{z}{x}\right)^{m}\left(1-\frac{z}{x}\right)^{n-m} e^{z-x} d x / \sum_{r=0}^{n-m}\left(\begin{array}{c}
n-m \\
r
\end{array}\right) \frac{z^{m+r}}{m+r-1) !} .
\end{aligned}
$$

For this particular series and transformation we are able to express the remainder as an integral divided by a finite sum. A computer programme using equation (2) and a table of $E_{1}(z)$ can be used to calculate numerical values of the remainder for small values of $m$ and $n$ and approximations to equation (4) may be used for larger values of $n$.

\section{Numerical values of the remainder}

In order to illustrate how the remainder is affected by transforming a series some values of the remainder for small values of $m$ and $n$ are given in Tables 1 and 2 . The leading diagonal consists of the remainders $R_{m, m}(z)$ for the original series while the parallel diagonals are the remainders after successive transformations of the series. The most important feature of these diagonals is that the remainders 
have a minimum at $m=|z|$ for the leading diagonal and $m=|z|+1$ for the other diagonals. However, this feature of the table is somewhat masked by the fact that the remainders decrease both when we increase $n$ and when we decrease $m$ to its minimum possible value, namely $m=1$. Finally, we note that the rate of convergence of the weighted Euler transformation is different in the two tables.

These observations may be extended by examining the integral for the remainder.

TABLE 1

Values of $R_{m, n}$ (2) calculated from $F_{1}(2)$ and $T_{m, n}(2)$

\begin{tabular}{rrrrrrr}
\hline$n$ & 1 & 2 & 3 & \multicolumn{1}{c}{4} & \multicolumn{1}{c}{5} & \multicolumn{1}{c}{6} \\
\hline 1 & -.13867 & .02800 & .00419 & .00086 & .00022 & .0000630 \\
2 & & .11133 & -.01367 & -.00231 & -.00051 & -.00014 \\
3 & & & -.13867 & .01133 & .00195 & 0.00044 \\
4 & & & & .23633 & -.01367 & -.00231 \\
5 & & & & & -.51367 & .02204 \\
6 & & & & & & 1.36133 \\
\hline
\end{tabular}

TABLE 2

Values of $R_{m, n}(-1+i)$ calculated from $F_{1}(-1+i)-T_{m, n}(-1+i)$

\begin{tabular}{|c|c|c|c|c|c|}
\hline$n^{n} \quad 1$ & 2 & 3 & 4 & 5 & 6 \\
\hline $\begin{array}{ll}1 & .383-196 i \\
2 & \\
3 & \\
4 & \\
5 & \end{array}$ & $\begin{array}{r}-.117+.304 i \\
.383+.304 i\end{array}$ & $\begin{array}{r}.133+.054 i \\
-.117-.196 i \\
-.117+.804 i\end{array}$ & $\begin{array}{r}.056-.061 i \\
-.117+054 i \\
.183-.096 i \\
-1.617+.804 i\end{array}$ & $\begin{array}{r}-.019-.050 i \\
.004+.074 i \\
-.002-.119 i \\
.183+.204 i \\
-4.617-2.196 i\end{array}$ & $\begin{array}{r}-.035-.007 i \\
.041+.022 i \\
.056-.061 i \\
.139-.001 i \\
-.206+.451 i \\
-4.617-17.196 i\end{array}$ \\
\hline
\end{tabular}

\section{Approximations to the remainder}

We now calculate an approximation to the remainder by using equation (4), Stirling's approximation for the factorials and saddle point approximations to the integrals. The numerator can be written in the form

$$
\int_{z}^{\infty} e^{f(x)} d x \text { where } f(x)=z-x+m \ln z+(n-m) \ln (x-z)-n \ln x .
$$

The integrand has two saddle points. If $z$ does not lie on the negative real axis, the saddle point appropriate to the integral from $z$ directly to infinity is $x_{0}$, where

$$
x_{0}=\frac{1}{2}\left[z-m+\sqrt{ }\left\{(z-m)^{2}+4 n z\right\}\right] \text {. }
$$

The sign of the square root is chosen so that the imaginary parts of $z$ and $x_{0}$ have the same sign. 
The saddle point approximation to the integral is then

$$
e^{z-x_{0}} \frac{z^{m}\left(x_{0}-z\right)^{n-m+1}}{x_{0}^{n-1}} \frac{\sqrt{ }(2 \pi)}{\sqrt{\left\{(n-m) x_{0}^{2}-n\left(x_{0}-z\right)^{2}\right\}}} .
$$

If $n$ is large this may be further approximated as $\sqrt{ }(\pi) z^{+n+1} n^{-1 m+1} \exp \left\{-2 \sqrt{ }(n z)+\frac{1}{2} z+\left(-\frac{1}{3} z^{2}+2 m z+m^{2}-2 m\right) / 4 \sqrt{ }(n z)+O(1 / n)\right\}$.

The denominator of equation (4) may also be approximated by an integral

$$
\int_{-\mathbf{l}}^{n-m+1}\left(\begin{array}{c}
n-m \\
r
\end{array}\right) \frac{z^{m+r}}{(m+r-1) !} d r
$$

If $n$ is sufficiently large that $|z|$ lies in the range $m /(n-m)<|z|<(n-m)(n-1)$ the integrand is small at the ends of the range and has a single maximum so we may again use a saddle point approximation.

As before, one of the two saddle points is near the path of integration giving a saddle point approximation for the denominator of equation (4)

$$
\frac{(n-m) ! e^{n+r+1}\left(r+\frac{1}{2}\right)^{m}\left(r+m-\frac{1}{2}\right)}{2 \pi\left(n-r-m+\frac{1}{2}\right)^{n} \sqrt{\left\{n(n-m+1)-\left(n-r-m+\frac{1}{2}\right)^{2}\right\}}},
$$

where

$$
r=\frac{1}{2}\left[\sqrt{ }\left\{4 z n+(z-m+1)^{2}\right\}-z-m\right] .
$$

If $n$ is large this may be further approximated as

$$
\begin{aligned}
& (2 \sqrt{\pi})^{-1} z^{1 m+\frac{1}{2}} n^{-1 m+t} \\
& \quad \times \exp \left\{2 \sqrt{ }(n z)-\frac{1}{2} z+\left(\frac{1}{3} z^{2}-2 m z-m^{2}+2 m-1\right) / 4 \sqrt{ }(n z)+O(1 / n)\right\}
\end{aligned}
$$

and the transformed remainder $R_{m, n}(z)$ is the ratio of the expressions (6) and (8) namely

$$
\begin{aligned}
& R_{m, n}(z)=(-1)^{m+1} 2 \pi \\
& \quad \times \exp \left\{-4 \sqrt{ }(n z)+z+\left(-\frac{1}{3} z^{2}+2 m z+m^{2}-2 m+\frac{1}{2}\right) / 2 \sqrt{ }(n z)+O(1 / n)\right\} .
\end{aligned}
$$

As an alternative to equation (9) if we are concerned with a sequence of transformations of the same order, $k=n-m$, and $k$ is large then equation (9) may be rewritten as

$$
\begin{aligned}
& R_{m, m+k}(z)=(-1)^{m-1} 2 \pi \\
& \quad \times \exp \left\{-4 \sqrt{ }(k z)+z+\left(-\frac{1}{3} z^{2}-2 m z+m^{2}-2 m+\frac{1}{2}\right) / 2 \sqrt{ }(k z)+O(1 / k)\right\} .
\end{aligned}
$$

As a check on the accuracy of the approximations used we evaluate $R_{1,6}(2)$; (i) exactly .0000630 , (ii) from expressions (5) and (7) .0000605 , (iii) from expression (9) .0000609 , (iv) from equation (10) .0000593 , and these improve as $n$ increases. 


\section{Three limiting properties of the weighted Euler transformation}

I. If $z$ is not on the negative real axis then $R_{m, n}(z) \rightarrow 0$ as $n \rightarrow \infty$.

Proof. If in equation (9) $m$ and $z$ are constant and $n$ is large the dominant term is $e^{-4 \sqrt{ }(n z)}$, and if $z$ is not on the negative real axis then $\sqrt{ } z$ has a positive real part, so $e^{-4 \sqrt{ }(n z)} \rightarrow 0$ as $n \rightarrow \infty$. Hence $R_{m, n}(z) \rightarrow 0$ as $n \rightarrow \infty$.

Other related properties are: (i) if $z$ is real negative then $\sqrt{ }(n z)$ will be pure imaginary; hence as $n$ increases the phase of $e^{-4 \sqrt{ }(n z)}$ will change but its amplitude will not decrease and so $R_{m, n}(z)$ will, to this approximation, not decrease if $z$ is on the negative real axis; (ii) the rate of decay of $R_{m, n}(z)$ as $n$ increases depends on the value of $e^{-4 \sqrt{ }\} n \mathscr{R}(\sqrt{ } z)\}}$. If $z=\xi+i \eta$ then $\mathscr{R}(\sqrt{ } z)=\sqrt{ }\left\{\xi+\sqrt{ }\left(\xi^{2}+\eta^{2}\right)\right\}$ which is constant on a parabola with focus at the origin and enclosing the negative real axis. Hence the values of $z$ which give the same rate of decay as $n$ increases lie on a parabola in the complex plane with focus at the origin. However, the actual size of $R_{m, n}(z)$ depends also on the other terms in the exponential in equation (9), and in particular on the factor $e^{z}$ whose modulus depends on the real part of $z$.

II. If $n$ and $z$ are constant the remainder may be reduced by decreasing $m$ to 1 .

Proof. If $n$ and $z$ are constant and $z=(p+i q)^{2}$, then equation (9) may be written in the form

$$
\begin{aligned}
& K \exp \left[\frac{1}{2\left(p^{2}+q^{2}\right) \sqrt{(n)}}\right. \\
& \left.\quad \times\left\{p\left(m^{2}-2 m+2 m\left(p^{2}+q^{2}\right)\right)+i q\left(-m^{2}+2 m+2 m\left(p^{2}+q^{2}\right)\right)\right\}+O(1 / n)\right],
\end{aligned}
$$

where $K$ is a complex constant. $\sqrt{z}$ maps onto the right-hand half $x$ plane so $p$ is positive and so the real part of the exponential increases if $m>1-p^{2}-q^{2}$ and the minimum value of $m$ is 1 . Hence, the remainder may be reduced by decreasing $m$ to 1 .

III. The sequence of remainders for all transformations of order $k$ has a minimum when $m=|z|+1+O(1 / \sqrt{ } k)$.

Proof. If $k$ and $z$ are constants and $z=(p+i q)^{2}$ then equation (10) may be written in the form

$$
\begin{aligned}
K \exp [ & \frac{1}{2\left(p^{2}+q^{2}\right) \sqrt{(k)}} \\
& \times\left\{p\left(m^{2}-2 m-2 m\left(p^{2}+q^{2}\right)+i q\left(-m^{2}+2 m-2 m\left(p^{2}+q^{2}\right)\right)\right\}+O(1 / k)\right],
\end{aligned}
$$


where $K$ is a complex constant and $p$ is positive. For a minimum remainder we minimize the real part of the exponential by choosing $m=1+p^{2}+q^{2}=1+|z|$, the terms neglected being of order $\sqrt{ } k$ smaller than those considered. As a comment we should compare this result with the minima for small $m$ and $n$ in Tables 1 and 2 .

\section{Extension to more general series}

We now consider a more general theorem but restrict ourselves to alternating series of real terms.

Let $\Sigma(-1)^{r} f_{r}(x)$ be an alternating series of real terms which is an asymptotic expansion of a function $F(x)$ whose remainder after $n$ terms is $(-1)^{n} f_{n}(x) \lambda(n, x)$.

THEOREM. If $f_{n}(x)$ is positive and $\Delta^{n-m} \lambda(m, x)$ is bounded, where

$$
\Delta \lambda(m, x)=\lambda(m+1, x)-\lambda(m, x),
$$

then the remainder of the asymptotic expansion may be made as small as we please by a weighted Euler transformation.

Proof. The remainder after a weighted Euler transformation using binomial coefficients and the reciprocals of the last terms as weights is

$$
\sum_{r=m}^{n}(-1)^{r}\left(\begin{array}{l}
n-m \\
r-m
\end{array}\right) \lambda(m+r, x) / \sum_{r=m}^{n}\left(\begin{array}{l}
n-m \\
r-m
\end{array}\right) \frac{1}{f_{r}(x)} .
$$

The numerator is $(-1)^{n} \Delta^{n-m} \lambda(m, x)$. Also, all the terms of the denominator are positive and if $m<k<n$ then a subset of these terms is

$$
\frac{(n-m) !}{(k-m) !(n-k) !} \frac{1}{f_{k}(x)} \text {. }
$$

If $k>m$ and $k$ and $m$ are held constant while $n$ is increased then the denominator is greater than $(n-m) ! /(k-m) !(n-k) ! f_{k}(x)$ which tends to infinity as $n$ is increased. Hence, if the numerator is bounded, the remainder may be made as small as we please by a weighted Euler transformation.

\section{Transforming Taylor's series}

The Taylor's series for $f(x)$ with the integral form of remainder is

$$
f(x)=\sum_{s=0}^{n} \frac{(x-a)^{s}}{s !} f^{(s)}(a)+\int_{a}^{x} \frac{(x-t)^{n}}{n !} f^{(n+1)} d t .
$$

If we apply a weighted Euler transformation to a set of these series weighting the set of series of $(m+1)$ up to $(n+1)$ terms inclusive with the binomial coefficients and reciprocals of the last term of each series while alternating their 
signs, namely

$$
(-1)^{r}\left(\begin{array}{l}
n-m \\
r-m
\end{array}\right) \frac{r !}{(x-a)^{r} f^{(r)}(a)}
$$

then the transformed remainder is

$$
\sum_{r=m}^{n}(-1)^{r}\left(\begin{array}{l}
n-m \\
r-m
\end{array}\right) \int_{a}^{x} \frac{(x-t)^{r}}{(x-a)^{r}} \frac{f^{(r+1)}(t)}{f^{(r)}(a)} d t / \sum_{r=m}^{n}(-1)^{r}\left(\begin{array}{l}
n-m \\
r-m
\end{array}\right) \frac{r !}{(x-a)^{r} f^{r)}(a)}
$$

A similar expression for the remainder may also be obtained as in Section 2(ii) using the next terms of the Taylor's series in the weights.

If the Taylor's series satisfies the conditions for the theorem of Section 7 , namely that the numerator is bounded for all $n$ and the terms of the denominator are all positive, then the transformed remainder has a zero limit as $n \rightarrow \infty$. If these conditions apply outside the original radius of convergence of the Taylor's series then the transformation will give $f(x)$ outside the radius of convergence.

\section{References}

M. Abramowitz and I. A. Stegun (1964), Handbook of Mathematical Functions (National Bureau of Standards, Appl. Math. Series), 55.

J. E. Drummond (1972), "A formula for accelerating the convergence of a general series, Bull. Austral. Math. Soc. 6, 69-74.

D. Shanks (1955), "Non-linear transformations of divergent and convergent sequences", J. Math. Phys. 34, 1-42.

L. Sirovich (1971), Techniques of Asymptotic Analysis, App. Math. Sc. 2 (Springer-Verlag).

Department of Applied Mathematics

Australian National University

Canberra

Australia 